\title{
Utilisation optimale de ressources alimentaires localement disponibles pour l'engraissement des ovins au sein des exploitations mixtes agriculture-élevage du plateau central du Burkina Faso
}

\author{
J.S. Zoundi ${ }^{1 *}$ A.J. Nianogo ${ }^{2}$ L. Sawadogo ${ }^{3}$
}

\begin{abstract}
Mots-clés
Ovin Djallonké - Engraissement Aliment de substitution - Urée Piliostigma reticulatum - Analyse coût avantage - Burkina Faso.
\end{abstract}

\begin{abstract}
Résumé
Deux essais ont été effectués avec des ovins Djallonké à l'engrais dans le but de rechercher des systèmes d'alimentation pour la production de viande à moindre coût. Dans cette étude ont été examinées les possibilités de substitution partielle ou totale d'un concentré commercial " Aliment Kibsa » (Ksa) (17,60 p. 100 de matières azotées digestibles; 0,91 UF/kg de matière sèche) ou du tourteau de coton (TC) par des sources énergétiques et azotées disponibles localement. Une ration témoin (R1) [40 p. 100 paille de sorgho (PS) + 60 p. $100 \mathrm{Ksa}$ a ainsi été comparée à différentes combinaisons alimentaires intégrant des produits locaux : dans R2 il y a eu une substitution partielle de Ksa (- 30 p. 100) par 27,50 p. 100 de gousses de Piliostigma reticulatum (GPr) + urée (Ur) et dans R3 il y a eu une substitution totale de Ksa par 47 p. 100 de $\mathrm{TC}+\mathrm{GPr}+$ Ur. Les gains moyens quotidiens (gmq) enregistrés dans I'essai 1 ont présenté des différences significatives $(P<0,01)$ avec 100,5, 62,4 et 58,6 g respectivement pour R1, R2 et R3. Ces substitutions se sont traduites par des pertes respectives en gmq de $-38,1$ et $-49,2 \mathrm{~g}$. Toutefois, R2 et R3 ont présenté les meilleurs profits, avec respectivement des bénéfices nets additionnels de +519 et +1055 Fcfa par animal. L'essai 2 a montré que la substitution partielle de TC (- 10 p. 100) par du foin de Pennisetum pedicellatum (Pp) s'est traduite par des pertes en gmq de $-14,4 \mathrm{~g}$. En conclusion, la combinaison entre 20-30 p. 100 de TC ou de Ksa et 27 p. 100 de GPr + Ur a permis, d'une part, une bonne valorisation des fourrages (PS ou Pp) et, d'autre part, aux producteurs d'enregistrer les meilleurs gains économiques.
\end{abstract}

\section{INTRODUCTION}

La conduite du petit élevage au sein des exploitations mixtes agriculture-élevage constitue une activité particulièrement stratégique pour les producteurs (12) en tant que source de liquidité, d'assurance contre les risques en agriculture pluviale, de fertilisants organiques pour les cultures, d'épargne sur pied... Toutefois, l'engrais-

\footnotetext{
1. Inera, 04 BP 8645, Ouagadougou 04, Burkina Faso

2. Université polytechnique, 01 BP 1091, Bobo-Dioulasso 01, Burkina Faso

3. Fast-UO, 03 BP 7021, Ouagadougou 03, Burkina Faso

* Auteur assurant la correspondance

Tél. : (226) 340270 ou 347112 ; fax : (226) 340271

E-mail : zoundi@hotmail.com ou zoundi@fasonet.bf
}

sement saisonnier des ovins, bien que constituant une opportunité de mobilisation de revenus en saison sèche, connaît de sérieuses contraintes d'alimentation, plus particulièrement celle relative à l'accès des producteurs aux concentrés produits hors ferme.

Ces problèmes de stratégie d'alimentation des animaux au sein des systèmes mixtes ont été à la base de plusieurs travaux de recherche pour permettre une meilleure valorisation de produits de substitution et contribuer ainsi à la réduction des coûts d'alimentation. Ces investigations ont été orientées vers divers sujets, parmi lesquels les systèmes de complémentation à base d'aliments non-conventionnels $(15,20)$, les traitements chimiques de la paille $(6,19)$, la valorisation de la croissance compensatrice par le biais de la complémentation stratégique (29), les systèmes de rationnement à partir des résidus de culture (23). D'autres investigations ont mis en évidence la possibilité de valoriser des fourrages pauvres par 
l'utilisation de ressources azotées et énergétiques disponibles localement $(8,27,29)$. Pour optimiser l'utilisation des sous-produits agro-industriels, des essais (17) ont permis la formulation d'un concentré dénommé Kibsa (Ksa) [30 p. 100 de tourteau de coton (TC), 27 p. 100 de son de blé, 26 p. 100 de graines de coton, 14 p. 100 de mélasse ou de poudre de néré, 1,70 p. 100 de coquilles d'huîtres et 1,30 p. 100 de sel], avec une ration retenue pour l'embouche intensive comprenant 40 p. 100 de paille de sorgho (PS) et 60 p. 100 de Ksa.

Tenant compte du contexte socio-économique des exploitations mixtes du plateau central du Burkina Faso, marqué notamment par la faible disponibilité en ressources financières, la présente étude a examiné les possibilités de valorisation des fourrages pauvres pour la production de viande à moindre coût à travers l'utilisation raisonnée de sources azotées protéiques et non-protéiques, et énergétiques disponibles à la ferme. Dans l'essai 1 ont été analysées les possibilités de substitution partielle du concentré Ksa par des sources azotées et énergétiques localement disponibles, et dans l'essai 2 celles liées à la substitution partielle de tourteau de coton par l'utilisation combinée de fourrages produits au sein de la ferme et de sources énergétiques et azotées facilement accessibles aux producteurs.

\section{MATERIEL ET METHODES}

Deux essais consécutifs, l'un en milieu réel (essai 1) et l'autre en station (essai 2) ont été conduits avec des ovins mâles entiers de race Djallonké. Tous les animaux ont été vaccinés contre la pasteurellose ovine et déparasités avant le démarrage de chaque essai. Les pesées ont lieu eu une fois par semaine à $7 \mathrm{~h} 00$ du matin après 12 heures de jeûne. Des mesures d'azote ammoniacal $\left(\mathrm{N}-\mathrm{NH}_{3}\right) \mathrm{du}$ jus de rumen ont été effectuées en deux prises journalières ( $10 \mathrm{~h} 00$ et $18 \mathrm{~h} 00)$ et quatre heures après la distribution des aliments selon les procédures de prélèvement décrites par Preston et Leng (20). Le dosage du N-NH $\mathrm{NH}_{3}$ a été fait selon la méthode de Conway (9).

Chaque essai a duré 112 jours y compris une période d'adaptation de 14 jours. Les aliments ont été offerts sur la base de $2,5 \mathrm{~kg}$ de matière sèche (MS) pour $100 \mathrm{~kg}$ de poids vif, lequel taux a été progressivement réajusté jusqu'à 3,5 p. 100 en fin d'expérimentation. Les aliments grossiers [PS et foin de Pennisetum pedicellatum Trin. (Pp)] ont été fournis en limitant les refus à un taux de 10-15 p. 100. A la fin de chaque essai, un échantillon de quatre animaux par lot a été abattu et les analyses des performances d'habillage et de découpe des carcasses ont été effectuées selon les procédures décrites par Boccard et Dumont $(4,5)$.

L'essai 1 a concerné des ovins âgés de 14-17 mois et de poids vif initial de 19,3 $\pm 2,6 \mathrm{~kg}$. Deux traitements expérimentaux (tableau I) ont été étudiés en comparaison avec le témoin incorporant le concentré Ksa [17,6 p. 100 de matières azotées digestibles (MAD); $0,91 \mathrm{UF} / \mathrm{kg}$ MS] dans un dispositif en blocs incomplets avec deux répétitions (atelier d'embouche ou bloc) par traitement ; chaque atelier d'embouche comportant 10 animaux, soit un total de 70 ovins.

Tenant compte de l'objectif de l'étude, un mélange de poudre de gousses de Piliostigma reticulatum (DC.) Hochst (GPr) (5,8 p. 100 MAD ; 0,41 UF/kg MS), aliment traditionnellement utilisé par les producteurs, et d'urée (Ur) a été utilisé en substitution partielle au concentré. Ce complément a été servi en quatre prises fractionnées dans la journée.

Pour l'essai 2, des ovins mâles entiers âgés de 12-15 mois et de poids vif initial de $16,8 \pm 0,5 \mathrm{~kg}$ ont été utilisés dans un dispositif factoriel à deux facteurs. Le facteur 1 a représenté la nature du
Tableau I

Dispositif expérimental (essai 1)

\begin{tabular}{lccc} 
& \multicolumn{3}{c}{ Traitements } \\
\cline { 2 - 4 } & $\begin{array}{c}\text { Témoin } \\
(\mathbf{6 0} \% \text { Ksa) })\end{array}$ & $\begin{array}{c}\text { Substitution } \\
\text { partielle de } \\
\text { Ksa par } \\
\text { GPr + Ur }\end{array}$ & $\begin{array}{c}\text { Substitution } \\
\text { totale de } \\
\text { de Ksa par } \\
\text { TC + GPr + Ur }\end{array}$ \\
\cline { 2 - 4 } & Ration 1 & Ration 2 & Ration 3 \\
\hline Composition (\%) & & & \\
PS & 40 & 40 & 50,50 \\
Ksa & 60 & 30 & - \\
TC & - & - & 20 \\
GPr & - & 26 & 26 \\
Ur & - & 1,50 & 1 \\
Pierre à lécher & & 2,50 & 2,50 \\
Valeur théorique ${ }^{a}$ & & & \\
MAD (\%) & 10,68 & 11,11 & 11,62 \\
UF/kg MS & 0,67 & 0,50 & 0,43 \\
Nombre d'ateliers & 2 & 2 & 3
\end{tabular}

PS : paille de sorgho ; Ksa : aliment Kibsa ; GPr : gousses de Piliostigma reticulatum ; Ur : urée ; TC : tourteau de coton

${ }^{a}$ Calcul effectué à partir des équations de régression pour la détermination des éléments digestibles totaux selon Harris (Rivière, 1991, Cirad-Iemvt)

MAD : matières azotées digestibles

fourrage de base : paille de sorgho (0,30 p. 100 MAD ; 0,30 UF/kg MS) ou foin de Pennisetum pedicellatum Trin. (Pp) (2,9 p. 100 $\mathrm{MAD} ; 0,39 \mathrm{UF} / \mathrm{kg} \mathrm{MS})$. Le facteur 2 a représenté la source azotée : tourteau de coton (TC) (35,2 p. 100 MAD ; 1,04 p. $100 \mathrm{UF} / \mathrm{kg}$ MS) ou foin de niébé (FN) (9,2 p. 100 MAD ; 0,60 UF/kg MS). Un total de quatre traitements expérimentaux (tableau II) ont été évalués en comparaison avec le témoin identique à celui de l'essai 1 , chaque traitement comportant 10 animaux, soit un total de 50 ovins. La formulation des rations a été faite de manière à permettre des comparaisons deux à deux (ration 2 et ration 4 ; ration 3 et ration 5) avec pour objectif de voir si l'amélioration de la qualité $\mathrm{du}$ fourrage de base pouvait se traduire par une économie en sources azotées ainsi que des gains économiques plus intéressants pour les producteurs.

Les évaluations économiques des deux essais ont été basées sur l'analyse coût-avantage selon les procédures décrites par Amir et Knipscheer (3). Les analyses statistiques des données ont été effectuées à l'aide des procédures de SAS (22) et selon le test de Newman-Keuls.

\section{RESULTATS ET DISCUSSION}

\section{Essai 1}

\section{Performances pondérales et consommation alimentaire}

Les valeurs des gains moyens quotidiens (gmq) obtenus (tableau III) ont été de 100,5, 62,4 et 58,6 g, respectivement pour les rations 1,2 et 3 . Les valeurs de gmq obtenues avec le témoin (ration 1) ont été comparables à celles obtenues par Nianogo et coll. (19). Le gmq moyen enregistré avec la ration 3 a été, quant à lui, légèrement inférieur à celui obtenu $(64,1 \mathrm{~g})$ par Zoundi et coll. (28) sur 


\section{Tableau II}

Dispositif expérimental (essai 2)

\begin{tabular}{|c|c|c|c|c|c|}
\hline & \multicolumn{5}{|c|}{ Traitements } \\
\hline & Témoin (60 \% Ksa) & $\begin{array}{c}\text { PS + } 20 \% \text { TC } \\
+27 \% \text { GPr + Ur }\end{array}$ & $\begin{array}{c}\text { PS }+40 \% \text { FN } \\
+13 \% \mathrm{GPr}+\mathrm{Ur}\end{array}$ & $\begin{array}{c}\mathrm{Pp}+10 \% \mathrm{TC} \\
+27,50 \% \mathrm{GPr}+\mathrm{Ur}\end{array}$ & $\begin{array}{c}\mathrm{Pp}+30 \% \mathrm{FN} \\
+13 \% \mathrm{GPr}+\mathrm{Ur}\end{array}$ \\
\hline & Ration 1 & Ration 2 & Ration 3 & Ration 4 & Ration 5 \\
\hline \multicolumn{6}{|c|}{ Composition (\%) } \\
\hline PS & 40 & 50,50 & 45,50 & - & - \\
\hline $\mathrm{Pp}$ & - & - & - & 60 & 54,50 \\
\hline Ksa & 60 & - & - & - & - \\
\hline TC & - & 20 & - & 10 & - \\
\hline FN & - & - & 40 & - & 30 \\
\hline GPr & - & 26 & 12 & 26 & 12 \\
\hline Ur & - & 1 & 1 & 1,50 & 1 \\
\hline Pierre à lécher & - & 2,50 & 2,50 & 2,50 & 2,50 \\
\hline \multicolumn{6}{|c|}{ Valeur théorique ${ }^{a}$} \\
\hline MAD (\%) & 10,68 & 11,36 & 7,26 & 10,84 & 7,8 \\
\hline UF/kg MS & 0,67 & 0,42 & 0,42 & 0,43 & 0,44 \\
\hline
\end{tabular}

PS : paille de sorgho ; Ksa : aliment Kibsa ; GPr : gousses de Piliostigma reticulatum ; Ur : urée ; TC : tourteau de coton ; FN : foin de niébé ;

$\mathrm{Pp}$ : foin de Pennisetum pedicellatum

${ }^{a}$ Calcul effectué à partir des équations de régression pour la détermination des éléments digestibles totaux selon Harris (Rivière, 1991, Cirad-Iemvt)

MAD : matières azotées digestibles

\section{Tableau III}

Performances pondérales et consommation volontaire des animaux (essai 1)

\begin{tabular}{|c|c|c|c|c|c|}
\hline & \multicolumn{3}{|c|}{ Traitements } & \multirow{3}{*}{$\begin{array}{c}\text { Signification } \\
\text { effets } \\
\text { traitements }\end{array}$} & \multirow{3}{*}{ Ecart-type } \\
\hline & $\begin{array}{l}\text { Témoin } \\
(60 \% \mathrm{Ksa})\end{array}$ & $\begin{array}{c}\text { Substitution } \\
\text { partielle Ksa } \\
\text { par GPr + Ur }\end{array}$ & $\begin{array}{l}\text { Substitution } \\
\text { totale de Ksa par } \\
\text { TC + GPr + Ur }\end{array}$ & & \\
\hline & Ration 1 & Ration 2 & Ration 3 & & \\
\hline \multicolumn{6}{|l|}{ Poids vif (kg) } \\
\hline Semaine 0 & $19,4^{\mathrm{a}}$ & $19,3^{\mathrm{a}}$ & $19,3^{a}$ & NS & 3,04 \\
\hline Semaine 4 & $22,3^{a}$ & $20,5^{\mathrm{a}}$ & $20,9^{a}$ & NS & 3,20 \\
\hline Semaine 8 & $24,9^{a}$ & $22,3^{b}$ & $22,7^{b}$ & $* *$ & 3,39 \\
\hline Semaine 12 & 27,8 & $24,5^{b}$ & $24,2^{b}$ & $* * *$ & 3,52 \\
\hline Gain total de poids vif & $8,4^{\mathrm{a}}$ & $5,2^{b}$ & $4,9^{b}$ & $* * *$ & 1,59 \\
\hline \multicolumn{6}{|l|}{ Gain moyen quotidien (g) } \\
\hline Semaines 1-4 & $102,7^{\mathrm{a}}$ & $43,1^{b}$ & $59,1^{b}$ & $* * *$ & 43,0 \\
\hline Semaines 5-8 & $95,7^{\mathrm{a}}$ & $65,0^{\mathrm{b}}$ & $64,0^{b}$ & $* * *$ & 32,40 \\
\hline Semaines 9-12 & $103,2^{\mathrm{a}}$ & $78,9^{b}$ & $52,6^{c}$ & $* * *$ & 26,67 \\
\hline Semaines 1-12 & $100,5^{\mathrm{a}}$ & $62,4^{b}$ & $58,6^{\mathrm{C}}$ & $* * *$ & 18,52 \\
\hline \multicolumn{6}{|l|}{ Consommation alimentaire } \\
\hline Ingestion volontaire (g MS/kg $\mathrm{P}^{0,75}$ ) & $72,7^{\mathrm{a}}$ & $68,4^{b}$ & $70,9^{b}$ & $* * *$ & 1,15 \\
\hline \multicolumn{6}{|l|}{ Indice de consommation } \\
\hline $\mathrm{Kg} \mathrm{MS} / \mathrm{kg}$ de gain & $8,0^{b}$ & $11,0^{\mathrm{a}}$ & $13,1^{\mathrm{a}}$ & $* * *$ & 3,20 \\
\hline UF/kg gain & $6,1^{\mathrm{a}}$ & $6,2^{\mathrm{a}}$ & $5,7^{b}$ & $* * *$ & 0,27 \\
\hline $\mathrm{N}-\mathrm{NH}_{3}$ jus de rumen $(\mathrm{mg} / \mathrm{l})$ & $155,2^{\mathrm{a}}$ & $191,8^{\mathrm{a}}$ & $190,7^{\mathrm{a}}$ & NS & 17,62 \\
\hline \multicolumn{6}{|c|}{ PS : paille de sorgho ; Ksa : aliment Kibsa ; GPr : gousses de Piliostigma reticulatum ; Ur : urée ; TC : tourteau de coton } \\
\hline
\end{tabular}

$* * \mathrm{P}<0,05 ; * * * \mathrm{P}<0,01 ; \mathrm{NS}=$ non significatif 
la même race et avec une ration identique. Il a également été inférieur aux valeurs obtenues $(78,4 \mathrm{~g})$ par Nianogo et coll. (18) sur des ovins de même race en conduite semi-intensive (pâture libre en saison de pluies et complémentation à 25 p. 100 des besoins en MS avec un mélange 50 p. 100 TC + 50 p. 100 son de blé).

Des différences significatives $(\mathrm{P}<0,01)$ ont été observées entre les $\mathrm{gmq}_{\mathrm{S} 1-12}$ des trois traitements. Ainsi, la substitution partielle de Ksa par le mélange GPr + Ur s'est soldée par une baisse significative des gmq, qui sont passés de 100,5 g pour la ration 1 à $62,4 \mathrm{~g}$ pour la ration 2, bien que les deux traitements aient eu le même taux en

MAD. Cette situation a révélé, d'une part, l'importance de la source azotée et, d'autre part, l'effet déterminant de l'apport énergétique. La complémentation avec l'urée a permis d'avoir des niveaux de $\mathrm{N}_{-} \mathrm{NH}_{3}$ élevés avec 191,8 et 190,7 mg/l pour les rations 2 et 3. Ces niveaux ont été supérieurs au seuil de $150 \mathrm{mg} / \mathrm{l}$ recommandés (20) pour une dégradation convenable des fourrages pauvres, mais ils n'ont pas eu d'effet sur les gains de poids vif, sans doute à cause de la réduction en source protéique mais surtout de l'insuffisance d'apport d'énergie. Cette tendance a été similaire à celle observée par Garcia et coll. (11), examinant les effets d'une substitution partielle de TC par de l'urée sur la croissance des ovins.

La substitution totale de Ksa par le TC à 20 p. 100 en combinaison avec le mélange GPr + Ur s'est également traduite par des pertes significatives $(\mathrm{P}<0,01)$ en gains de poids $(-49,2 \mathrm{~g})$.

Les résultats obtenus sur la consommation volontaire ont été comparables à ceux de Bourzat et coll. (7). Ils ont été inférieurs à ceux

Tableau IV

Performances d'abattage des animaux (essai 1)

\begin{tabular}{|c|c|c|c|c|c|}
\hline & \multicolumn{3}{|c|}{ Traitements } & \multirow{3}{*}{$\begin{array}{c}\text { Signification } \\
\text { effets } \\
\text { traitements }\end{array}$} & \multirow{3}{*}{ Ecart-type } \\
\hline & $\begin{array}{l}\text { Témoin } \\
(60 \% \text { Ksa) }\end{array}$ & $\begin{array}{l}\text { Substitution } \\
\text { partielle Ksa } \\
\text { par GPr + Ur }\end{array}$ & $\begin{array}{c}\text { Substitution } \\
\text { totale de Ksa par } \\
\text { TC + GPr + Ur }\end{array}$ & & \\
\hline & Ration 1 & Ration 2 & Ration 3 & & \\
\hline \multicolumn{6}{|l|}{ Poids carcasse et rendements } \\
\hline Carcasse chaude (kg) & $11,8^{\mathrm{a}}$ & $11,4^{\mathrm{a}}$ & $10,6^{\mathrm{a}}$ & NS & 1,1 \\
\hline Carcasse après ressuyage (kg) & $11,0^{\mathrm{a}}$ & $10,6^{\mathrm{a}}$ & $9,9^{\mathrm{a}}$ & NS & 1,0 \\
\hline Rendement vrai (\%) & $44,9^{\mathrm{a}}$ & $43,63^{\mathrm{a}}$ & $42,6^{\mathrm{a}}$ & NS & 2,4 \\
\hline \multicolumn{6}{|l|}{ Organes internes $(\mathrm{kg})$} \\
\hline Foie & $0,45^{\mathrm{a}}$ & $0,41^{\mathrm{ab}}$ & $0,36^{b}$ & $* * *$ & 0,04 \\
\hline Cœur & $0,15^{\mathrm{a}}$ & $0,14^{\mathrm{a}}$ & $0,13^{\mathrm{a}}$ & NS & 0,01 \\
\hline Reins & $0,08^{\mathrm{a}}$ & $0,07^{\mathrm{ab}}$ & $0,07^{\mathrm{ab}}$ & $* * *$ & 0,005 \\
\hline Rate & $0,12^{\mathrm{a}}$ & $0,09^{a}$ & $0,09^{a}$ & NS & 0,02 \\
\hline Rumen & $0,58^{a}$ & $0,55^{\mathrm{a}}$ & $0,61^{\mathrm{a}}$ & NS & 0,03 \\
\hline Feuillet + caillette & $0,23^{\mathrm{a}}$ & $0,19^{b}$ & $0,18^{b}$ & $* * *$ & 0,02 \\
\hline Intestins & $1,39^{\mathrm{a}}$ & $1,27^{a}$ & $1,17^{\mathrm{a}}$ & NS & 0,15 \\
\hline Poumons + trachée & $0,33^{\mathrm{a}}$ & $0,30^{\mathrm{a}}$ & $0,30^{\mathrm{a}}$ & NS & 0,03 \\
\hline Total organes/carcasse chaude & $29,61^{\mathrm{a}}$ & $28,01^{\mathrm{a}}$ & $28,74^{\mathrm{a}}$ & NS & 2,34 \\
\hline \multicolumn{6}{|l|}{ Gras (kg) } \\
\hline Gras de toilette & $0,46^{\mathrm{a}}$ & $0,35^{\mathrm{a}}$ & $0,33^{\mathrm{a}}$ & NS & 0,18 \\
\hline Gras périrénal & $0,35^{\mathrm{a}}$ & $0,24^{\mathrm{a}}$ & $0,24^{\mathrm{a}}$ & NS & 0,13 \\
\hline \multicolumn{6}{|l|}{ Catégories morceaux } \\
\hline \multicolumn{6}{|l|}{ Catégorie 1} \\
\hline Poids (kg) & $5,8^{\mathrm{a}}$ & $5,4^{\mathrm{a}}$ & $5,1^{b}$ & $* * *$ & 1,17 \\
\hline En \% de la carcasse & $52,7^{\mathrm{a}}$ & $51,6^{\mathrm{a}}$ & $52,5^{\mathrm{a}}$ & NS & 0,27 \\
\hline \multicolumn{6}{|l|}{ Catégorie 2} \\
\hline Poids (kg) & $3,1^{\mathrm{a}}$ & $3,0^{\mathrm{a}}$ & $2,8^{\mathrm{a}}$ & NS & 0,89 \\
\hline En \% de la carcasse & $28,5^{b}$ & $29,1^{\mathrm{a}}$ & $28,8^{a}$ & $* * *$ & 0,22 \\
\hline \multicolumn{6}{|l|}{ Catégorie 3} \\
\hline Poids (kg) & $2,1^{\mathrm{a}}$ & $2,0^{\mathrm{a}}$ & $1,8^{\mathrm{a}}$ & NS & 1,25 \\
\hline En \% de la carcasse & $18,8^{a}$ & $19,2^{\mathrm{a}}$ & $18,7^{a}$ & NS & 0,057 \\
\hline
\end{tabular}

Ksa : aliment Kibsa ; GPr : gousses de Piliostigma reticulatum ; Ur : urée ; TC : tourteau de coton

Les valeurs figurant sur la même ligne et ne portant aucun indice commun sont significativement différentes selon le test de Newman-Keuls (Snk) pour : $* * * P<0,01$

NS = non significatif

Catégorie 1 : gigot, selle, filets, carré couvert

Catégorie 2 : carré découvert, épaules

Catégorie 3 : poitrine, collier 
de Tiendrebeogo (27) sur la race Bali Bali, mais supérieurs aux normes indiquées par Rivière (21). Les valeurs des indices de consommation ont été faibles comparées à celles obtenues par Tiendrebeogo (27) qui rapporte des indices allant de 14 à 23, mais supérieures aux valeurs obtenues par Amegee (2). Elles ont été comparables à celles observées par Bourzat et coll. (7), Dehoux et Hounsou-Ve (10) et Nianogo et coll. (19) et ont traduit une bonne efficacité de transformation alimentaire par les animaux, plus particulièrement ceux du traitement 3 .

\section{Performances d'abattage des animaux}

Les rendements des carcasses obtenus (tableau IV) ont été de 44,9, 43,6 et 42,6 p. 100 respectivement pour les rations 1,2 et 3 . Ces valeurs ont été proches de celles de Bourzat et coll. (7) et de Nianogo et coll. (19) (40-48 p. 100), mais elles ont été inférieures à celles obtenues par Amegee (2) (52-56 p. 100) et par Nianogo et coll. (18) (49-53 p. 100).

Concernant le développement des organes internes et hormis les reins et l'ensemble feuillet + caillette, il n'y a eu aucune différence significative entre les rations. Toutefois, un développement plus important d'organes internes des animaux utilisant la ration 1 a été observé. Cette situation qui traduit la relative disponibilité en nutriments dans la ration 1 a été similaire à celle observée chez des animaux soumis à la croissance compensatrice $(13,14,29)$.

\section{Analyse économique}

En termes de bénéfice net en liquidité, les résultats obtenus (tableau V) ont révélé un avantage des rations 2 et 3 (respectivement 3481 et 4017 Fcfa de bénéfice net par animal, contre 2962 Fcfa pour le témoin). Les marges brutes ont été dans la même fourchette de valeurs que celles obtenues par Tiendrebeogo (2 400 à 5200 Fcfa) (27), mais elles ont été inférieures aux valeurs reportées par Zoundi et coll. (4 000-5 200 Fcfa) (29) sur la même race. Toutefois, ces valeurs ont été largement supérieures à celles obtenues par Dehoux et Hounsou-Ve (910 Fcfa en moyenne par animal) (10) sur des béliers Djallonké en zone humide. En termes de renouvellement du capital investi, un net avantage des rations 2 et 3 a également été observé. Ces avantages économiques obtenus avec les traitements 2 et 3 malgré leurs faibles gains en poids (comparés au témoin) seraient dus non seulement aux coûts d'alimentation $(39,8$ p. 100 des charges totales de la ration 1 contre 23,7 et 18,1 p. 100 respectivement pour les rations 2 et 3 ), mais aussi à la «rente de situation » créée par la fête de la Tabaski et au

\section{Tableau V}

Analyse économique (en Fcfa par animal embouché) (essai 1)

\begin{tabular}{|c|c|c|c|}
\hline & \multicolumn{3}{|c|}{ Traitements } \\
\hline & Témoin (60\% Ksa) & $\begin{array}{l}\text { Substitution partielle } \\
\text { Ksa par GPr + Ur }\end{array}$ & $\begin{array}{c}\text { Substitution totale } \\
\text { de Ksa par TC + GPr + Ur }\end{array}$ \\
\hline & Ration 1 & Ration 2 & Ration 3 \\
\hline \multicolumn{4}{|l|}{ Coûts de production } \\
\hline \multicolumn{4}{|l|}{ Dépenses en liquidité } \\
\hline Aliments ${ }^{1}$ & 3525 & 1676 & 984 \\
\hline Animaux ${ }^{2}$ & 4790 & 4747 & 4754 \\
\hline Soins vétérinaires ${ }^{3}$ & 300 & 300 & 300 \\
\hline \multicolumn{4}{|l|}{ Dépenses en nature } \\
\hline Aliments 4 & 239 & 340 & 389 \\
\hline Coûts alimentation/charges (\%) & 39,8 & 23,7 & 18,1 \\
\hline \multicolumn{4}{|l|}{ Recettes } \\
\hline Recettes en liquidité ${ }^{5}$ & 11577 & 10204 & 10055 \\
\hline Recettes en nature ${ }^{6}$ & 126 & 126 & 126 \\
\hline \multicolumn{4}{|l|}{ Résultats } \\
\hline Marges brutes a & 2849 & 3278 & 3760 \\
\hline Bénéfice net en liquidité b & 2962 & 3481 & 4017 \\
\hline Rapport coût-avantage ${ }^{\mathrm{c}}$ & 1,32 & 1,46 & 1,87 \\
\hline Renouvellement capital d & 1,34 & 1,51 & 1,98 \\
\hline \\
\hline \multicolumn{4}{|c|}{${ }^{1}$ Prix $(\mathrm{Fcfa})$ par kilogramme de matière sèche $: \mathrm{Ur}=82 ; \mathrm{TC}=75 ; \mathrm{Ksa}=90 ;$ minéraux $=550$} \\
\hline \multicolumn{4}{|c|}{${ }^{2}$ Prix moyen d'achat des animaux : 247 Fcfa par kilogramme de poids vif } \\
\hline \multicolumn{4}{|l|}{${ }^{3}$ Coût moyen par animal : 300 Fcfa } \\
\hline \multicolumn{4}{|c|}{${ }^{4}$ Coût estimé $:$ paille de sorgho $=7 \mathrm{Fcfa} ; \mathrm{GPr}=8 \mathrm{Fcfa}$} \\
\hline \multicolumn{4}{|c|}{${ }^{5}$ Prix moyen réel à la vente : 416 Fcfa par kilogramme de poids vif } \\
\hline \multicolumn{4}{|c|}{${ }^{6}$ Fumier : $300 \mathrm{~g}$ par animal $/ \mathrm{j} ; 5$ Fcfa par kilogramme de fumier } \\
\hline \multicolumn{4}{|c|}{ a Recettes totales - coûts totaux } \\
\hline \multicolumn{4}{|l|}{${ }^{\mathrm{b}}$ Recettes en liquidité - dépenses en liquidité } \\
\hline \multicolumn{4}{|l|}{${ }^{\mathrm{c}}$ Recettes totales / coûts totaux } \\
\hline${ }^{\mathrm{d}}$ Recettes en liquidité / dépenses en liquidité & & & \\
\hline
\end{tabular}


mode de vente des animaux. En effet, pendant cette période, les prix de vente des ovins mâles sont particulièrement élevés et sont déterminés par d'autres critères (conformation, robe...) que le poids vifs. Un prix moyen réel de $416 \mathrm{Fcfa} / \mathrm{kg}$ de poids vif a été noté sur les marchés à partir de pesées effectuées pendant les ventes.

Les résultats ont montré que la substitution partielle de Ksa par le mélange GPr + Ur, même si elle s'est traduite par des gains de poids moindres, a permis aux producteurs d'avoir un bénéfice net en liquidité additionnel de + 519 Fcfa par animal engraissé. L'utilisation du mélange GPr + Ur en combinaison avec le TC à une plus faible proportion ( 20 p. 100 au lieu de 30 p. $100 \mathrm{Ksa}$ ) a constitué une alternative intéressante avec le gain économique en liquidité le plus élevé (gain additionnel de $+1055 \mathrm{Fcfa}$ et de +536 Fcfa par animal en comparaison avec les rations 1 et 2 ).

\section{Essai 2}

\section{Performances pondérales et consommation volontaire}

Les gains de poids observés (tableau VI) ont été globalement inférieurs à ceux enregistrés au cours de l'essai 1. Cette situation serait due aux cas de diarrhées enregistrés pendant $96 \mathrm{~h}$ dans la $7^{\mathrm{e}}$ semaine de l'essai. Les gmq (semaines 1-12) ont présenté des différences très significatives $(\mathrm{P}<0,01)$ avec une supériorité du traitement témoin. Les gmq enregistrés avec les rations 3 et 5 ont été les plus bas (respectivement 5,0 et 8,6 g), comparables à ceux obtenus par Tamboura et Abou (24) avec des animaux de même race utilisant la paille de riz et recevant une complémentation sous forme de bloc mélasse-urée.

La comparaison entre les rations 2 et 4 a montré que la diminution du taux d'incorporation du TC (- 10 p. 100) au profit de Pp a entraîné des pertes en gmq $(-14,4 \mathrm{~g})$. L'amélioration de la qualité du fourrage de base n'a donc pas été suffisante pour compenser le manque en nutriments dû à la baisse du taux de TC, le raisonnement de la substitution étant basé sur le pourcentage de MS d'aliment. Les rations 2 et 4 ont eu sensiblement les mêmes valeurs énergétiques et cette situation indiquerait l'effet négatif de la réduction en source protéique dans la ration 4. Le mélange GPr + Ur, sensé améliorer la digestion globale de la ration à travers l'amélioration de l'environnement du rumen, n'a pas produit l'effet escompté en termes de gains de poids. Cette tendance rejoint les observations de certains auteurs (25) montrant que

Tableau VI

Performances pondérales et consommation alimentaire (essai 2)

\section{Traitements}

$\begin{array}{ccccc}\text { Témoin } & \mathrm{PS}+20 \% \mathrm{TC} & \mathrm{PS}+40 \% \mathrm{FN} & \mathrm{Pp}+10 \% \mathrm{TC} & \mathrm{Pp}+30 \% \mathrm{FN} \\ (60 \% \mathrm{Ksa}) & +27 \% \mathrm{GPr} & +13 \% \mathrm{GPr} & +27,50 \% \mathrm{GPr} & +13 \% \mathrm{GPr} \\ & +\mathrm{Ur} & +\mathrm{Ur} & +\mathrm{Ur} & +\mathrm{Ur}\end{array}$

$\begin{gathered}\text { Signification } \\ \text { effets } \\ \text { traitements }\end{gathered}$
type

\begin{tabular}{|c|c|c|c|c|c|c|c|}
\hline & Ration 1 & Ration 2 & Ration 3 & Ration 4 & Ration 5 & & \\
\hline \multicolumn{8}{|l|}{ Poids vifs (kg) } \\
\hline Semaine 0 & $16,6^{\mathrm{a}}$ & $17,4^{\mathrm{a}}$ & $16,4^{\mathrm{a}}$ & $17,3^{\mathrm{a}}$ & $17,7^{\mathrm{a}}$ & NS & 0,56 \\
\hline Semaine 4 & $19,9^{a}$ & $19,0^{\mathrm{a}}$ & $17,5^{\mathrm{a}}$ & $18,3^{\mathrm{a}}$ & $18,0^{\mathrm{a}}$ & NS & 0,93 \\
\hline Semaine 8 & $20,8^{a}$ & $18,9^{a b}$ & $16,2^{b}$ & $17,9 \mathrm{ab}$ & $17,4^{\mathrm{ab}}$ & $* *$ & 1,70 \\
\hline Semaine 12 & $22,4^{\mathrm{a}}$ & $19,7^{\mathrm{ab}}$ & $16,6^{\mathrm{b}}$ & $18,6^{\mathrm{b}}$ & $18,0^{b}$ & $* * *$ & 2,90 \\
\hline Gain total de poids vif & $5,9^{\mathrm{a}}$ & $2,4^{b}$ & $0,1^{b}$ & $1,2^{b}$ & $0,3^{b}$ & $* * *$ & 2,33 \\
\hline \multicolumn{8}{|l|}{ Gmg (g) } \\
\hline Semaines 1-4 & $122,2^{\mathrm{a}}$ & $55,4^{b}$ & $34,4^{b}$ & $28,6^{b}$ & $17,9^{b}$ & $* * *$ & 36,8 \\
\hline Semaines 5-8 & $33,7^{a}$ & $8,0^{\mathrm{ab}}$ & $29,0^{\mathrm{a}}$ & $4,4^{\mathrm{ab}}$ & $14,3^{b}$ & $* * *$ & 32,8 \\
\hline Semaines 9-12 & $57,1^{\mathrm{a}}$ & $28,0^{\mathrm{ab}}$ & $9,8^{b}$ & $24,6^{b}$ & $22,2^{b}$ & $* * *$ & 19,9 \\
\hline Semaines 1-12 & $71,0^{\mathrm{a}}$ & $30,7^{b}$ & $5,1^{\mathrm{c}}$ & $16,3^{b c}$ & $8,6^{c}$ & $* * *$ & 17,7 \\
\hline $\begin{array}{l}\text { Consommation alimentaire } \\
\text { Ingestion volontaire } \\
\text { (g MS/kg P }{ }^{0,75} \text { ) }\end{array}$ & $61,0^{\mathrm{a}}$ & $58,8^{\mathrm{b}}$ & $59,7^{b}$ & $58,7^{b}$ & $56,5^{b}$ & $* * *$ & 1,60 \\
\hline \multicolumn{8}{|l|}{ Indice de consommation } \\
\hline $\mathrm{Kg} \mathrm{MS} / \mathrm{kg}$ de gain & $8,4^{b}$ & $16,8^{b}$ & $57,4^{\mathrm{a}}$ & $48,7^{\mathrm{a}}$ & $42,1^{\mathrm{a}}$ & $* * *$ & 21,06 \\
\hline UF/kg de gain & $5,3^{e}$ & $7,2^{\mathrm{d}}$ & $40,5^{\mathrm{a}}$ & $13,5^{\mathrm{c}}$ & $25,1^{b}$ & $* * *$ & 1,06 \\
\hline $\mathrm{N}-\mathrm{NH}_{3}$ jus de rumen $(\mathrm{mg} / \mathrm{l})$ & $171,9^{a}$ & $253,6^{a}$ & $253,6^{\mathrm{a}}$ & $190,6^{a}$ & $187,9^{a}$ & NS & 32,84 \\
\hline
\end{tabular}

Ksa : aliment Kibsa ; PS : paille de sorgho ; TC : tourteau de coton ; GPr : gousses de Piliostigma reticulatum ; Ur : urée ; Pp : foin de Pennisetum pedicellatum ; FN : foin de niébé

Gmq : gain moyen quotidien

Ksa : aliment Kibsa ; GPr : gousses de Piliostigma reticulatum ; Ur : urée ; TC : tourteau de coton

Les valeurs figurant sur la même ligne et ne portant aucun indice commun sont significativement différentes selon le test de Newman-Keuls (Snk) pour :

$* * \mathrm{P}<0,05 ; * * * \mathrm{P}<0,01$

$\mathrm{NS}=$ non significatif

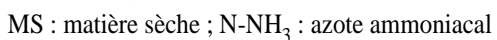


l'effet de la fourniture convenable de $\mathrm{N}^{-\mathrm{NH}_{3}}$ à travers l'apport en urée n'est maximisé qu'en présence d'une source protéique de bonne valeur nutritive.

La comparaison des rations 3 et 5 a montré un effet positif de la substitution, même s'il n'a pas été significatif. Cette tendance s'expliquerait par le fait que la substitution s'est traduite par un léger accroissement du niveau d'énergie et d'azote, rendant ainsi plus évident l'effet dû à l'amélioration de la qualité du fourrage de base. Malgré tout, les gains de poids enregistrés avec les rations 3 et 5 sont apparus faibles comparés à ceux des rations 2 et 4 , ce qui pose le problème de la disponibilité des sources énergétiques et azotées de bonne valeur nutritive au sein des exploitations.
Il y a donc un équilibre à rechercher entre sources énergétiques et azotées hors ferme de bonne valeur et celles disponibles localement ; certains auteurs $(1,16)$ aboutissent à des taux de substitution du TC par des fourrages azotés ligneux de 25 à 66 p. 100.

La consommation volontaire des aliments a donné des valeurs inférieures à celles enregistrées au cours de l'essai 1, mais avec la même tendance marquée par une supériorité du traitement témoin.

\section{Performances d'abattage des animaux}

Contrairement aux résultats de l'essai 1, le tableau VII montre des différences très significatives $(\mathrm{P}<0,01)$ pour les poids et les rendements des carcasses. D'une manière générale, les rendements

Tableau VII

Performances d'abattage des animaux (essai 2)

\begin{tabular}{|c|c|c|c|c|c|c|c|}
\hline & \multicolumn{5}{|c|}{ Traitements } & \multirow{3}{*}{$\begin{array}{c}\text { Signification } \\
\text { effets } \\
\text { traitements }\end{array}$} & \multirow{3}{*}{$\begin{array}{l}\text { Ecart- } \\
\text { type }\end{array}$} \\
\hline & $\begin{array}{l}\text { Témoin } \\
(60 \% \text { Ksa })\end{array}$ & $\begin{array}{c}\text { PS }+20 \% \text { TC } \\
+27 \% \text { GPr } \\
+ \text { Ur }\end{array}$ & $\begin{array}{c}\text { PS }+40 \% \text { FN } \\
+13 \% \text { GPr } \\
+ \text { Ur }\end{array}$ & $\begin{array}{c}\mathrm{Pp}+10 \% \mathrm{TC} \\
+27,50 \% \mathrm{GPr} \\
+\mathrm{Ur}\end{array}$ & $\begin{array}{c}\mathrm{Pp}+30 \% \mathrm{FN} \\
+13 \% \mathrm{GPr} \\
+\mathrm{Ur}\end{array}$ & & \\
\hline & Ration 1 & Ration 2 & Ration 3 & Ration 4 & Ration 5 & & \\
\hline \multicolumn{8}{|l|}{$\begin{array}{l}\text { Poids carcasse } \\
\text { et rendements }\end{array}$} \\
\hline Carcasse chaude (kg) & $10,4^{\mathrm{a}}$ & $7,9^{b}$ & $6,1^{\mathrm{c}}$ & $7,1^{\mathrm{bc}}$ & $7,0^{\text {bc }}$ & $* * *$ & 1,65 \\
\hline $\begin{array}{l}\text { Carcasse après } \\
\text { ressuyage }(\mathrm{kg})\end{array}$ & $10,1^{\mathrm{a}}$ & $7,5^{b}$ & $5,5^{c}$ & $6,7^{b c}$ & $6,7^{b c}$ & $* * *$ & 1,70 \\
\hline Rendement vrai (\%) & $42,7^{\mathrm{a}}$ & $37,0^{b}$ & $32,6^{\mathrm{c}}$ & $36,5^{\mathrm{bc}}$ & $36,0^{b c}$ & $* * *$ & 3,65 \\
\hline \multicolumn{8}{|l|}{ Organes internes (kg) } \\
\hline Foie & $0,37^{\mathrm{a}}$ & $0,26^{b}$ & $0,24^{b}$ & $0,26^{b}$ & $0,25^{b}$ & $* * *$ & 0,05 \\
\hline Cœur & $0,14^{\mathrm{a}}$ & $0,12^{\mathrm{ab}}$ & $0,09^{b}$ & $0,10^{b}$ & $0,09^{b}$ & $* * *$ & 0,02 \\
\hline Reins & $0,07^{\mathrm{a}}$ & $0,06^{\mathrm{a}}$ & $0,06^{\mathrm{a}}$ & $0,06^{\mathrm{a}}$ & $0,06^{\mathrm{a}}$ & NS & 0,004 \\
\hline Rate & $0,10^{\mathrm{a}}$ & $0,07^{a}$ & $0,06^{\mathrm{a}}$ & $0,06^{\mathrm{a}}$ & $0,07^{a}$ & NS & 0,02 \\
\hline $\begin{array}{l}\text { Rumen + feuillet } \\
+ \text { caillette }\end{array}$ & $0,78^{a}$ & $0,73^{a}$ & $0,67^{a}$ & $0,69^{a}$ & $0,75^{a}$ & NS & 0,04 \\
\hline Intestins & $1,20^{\mathrm{a}}$ & $0,92^{\mathrm{a}}$ & $0,80^{\mathrm{a}}$ & $0,88^{\mathrm{a}}$ & $0,80^{\mathrm{a}}$ & NS & 0,16 \\
\hline Poumons + trachée & $0,29^{a}$ & $0,26^{\mathrm{ab}}$ & $0,22^{b}$ & $0,27^{b}$ & $0,23^{b}$ & $* *$ & 0,03 \\
\hline $\begin{array}{l}\text { Total organes/ } \\
\text { carcasse chaude }\end{array}$ & $28,04^{b}$ & $30,16^{a b}$ & $34,72^{\mathrm{a}}$ & $32,22^{\mathrm{ab}}$ & $31,93^{a b}$ & $* *$ & 2,38 \\
\hline \multicolumn{8}{|l|}{ Gras (kg) } \\
\hline Gras de toilette & $0,40^{\mathrm{a}}$ & $0,07^{b}$ & $0,01^{b}$ & $0,04^{b}$ & $0,05^{b}$ & $* * *$ & 0,16 \\
\hline Gras périrénal & $0,28^{a}$ & $0,08^{b}$ & $0,02^{b}$ & $0,06^{b}$ & $0,05^{b}$ & $* * *$ & 0,10 \\
\hline \multicolumn{8}{|l|}{ Parties découpe en gros } \\
\hline Culotte (kg) & $3,1^{\mathrm{a}}$ & $2,4^{b}$ & $1,9^{\mathrm{c}}$ & $2,2^{b c}$ & $2,3^{b c}$ & $* * *$ & 0,24 \\
\hline $\begin{array}{l}\text { Culotte } \\
\text { (\% de la carcasse) }\end{array}$ & $32,1^{b}$ & $32,8^{\mathrm{ab}}$ & $34,9^{a}$ & $33,1^{\mathrm{ab}}$ & $35,0^{\mathrm{a}}$ & $* * *$ & 1,09 \\
\hline Coffre (kg) & $6,6^{\mathrm{a}}$ & $4,92^{b}$ & $3,6^{\mathrm{c}}$ & $4,4^{b}$ & $4,2^{\mathrm{bc}}$ & $* * *$ & 0,45 \\
\hline $\begin{array}{l}\text { Coffre } \\
\text { (\% de la carcasse) }\end{array}$ & $67,9^{\mathrm{a}}$ & $67,1^{\mathrm{a}}$ & $65,1^{\mathrm{b}}$ & $66,9^{\mathrm{ab}}$ & $65,0^{b}$ & $* * *$ & 1,10 \\
\hline
\end{tabular}

Ksa : aliment Kibsa ; PS : paille de sorgho ; TC : tourteau de coton ; GPr : gousses de Piliostigma reticulatum ; Ur : urée ; Pp : foin de Pennisetum pedicellatum ; FN : foin de niébé

Les valeurs figurant sur la même ligne et ne portant aucun indice commun sont significativement différentes selon le test de Newman-Keuls (Snk) pour :

$* * \mathrm{P}<0,05 ; * * * \mathrm{P}<0,01$

$\mathrm{NS}=$ non significatif

Culotte : gigots + selle

Coffre : épaules + collier + carrés (couvert et découvert) 
des carcasses sont apparus faibles, comparés aux valeurs obtenues par Amegee (2), Zoundi et coll. (28) et Nianogo et coll. (18).

Au niveau des organes internes, hormis les reins, la rate, l'estomac et les intestins, des différences significatives $(\mathrm{P}<0,05)$ ont également été observées. Comme pour l'essai 1, les plus fortes valeurs ont été enregistrées avec le traitement témoin. Toutefois, le rapport organes internes/carcasse chaude a montré des variations significatives $(\mathrm{P}<0,05)$, les valeurs les plus élevées ayant été observées avec les traitements expérimentaux. Cette tendance expliquerait en partie les faibles rendements des carcasses obtenus et rejoint les observations de Kabbali et coll. $(13,14)$ et de Zoundi et coll. (29), traduisant le fait que les nutriments sont prioritairement mobilisés au profit du développement des organes internes. Cette situation est non seulement liée à l'âge des animaux mais également à leur antécédent nutritionnel.

Les parties de la découpe en gros ont également présenté des variations significatives $(\mathrm{P}<0,01)$ et les tendances observées s'expliqueraient par l'effet du statut nutritionnel sur le développement différentiel des parties de la carcasse $(13,14,29)$.

\section{Analyse économique}

Les résultats consignés au tableau VIII montrent des bénéfices nets en liquidité variant de 3000 à 3700 Fcfa par animal. Les traitements expérimentaux ont enregistré les meilleurs gains économiques. Comme pour les gains de poids vif, la diminution du taux de TC au profit du Pp s'est traduite par une perte (- 324 Fcfa par animal en bénéfice net), alors que la substitution partielle du FN par le Pp s'est traduite par un gain (+ 309 Fcfa de bénéfice net par animal). Ces tendances observées s'expliquaient également par l'effet « rente de situation » créé par la fête de la Tabaski où d'autres critères que le poids vif sont intervenus dans la vente des animaux.

\section{CONCLUSION}

Cette étude a montré que les niveaux d'urée (1-1,5 p. 100) étaient appropriés pour assurer une production suffisante de $\mathrm{N}-\mathrm{NH}_{3}(\geq 150 \mathrm{mg} / \mathrm{l})$ nécessaire pour une utilisation convenable

\section{Tableau VIII}

Analyse économique (en Fcfa par animal embouché) (essai 2)

\begin{tabular}{|c|c|c|c|c|c|}
\hline & \multicolumn{5}{|c|}{ Traitements } \\
\hline & $\begin{array}{c}\text { Témoin } \\
(60 \% \mathrm{Ksa})\end{array}$ & $\begin{array}{c}\text { PS }+20 \% \text { TC } \\
+27 \% \text { GPr } \\
+ \text { Ur }\end{array}$ & $\begin{array}{c}\text { PS }+40 \% \text { FN } \\
+13 \% \mathrm{GPr} \\
+ \text { Ur }\end{array}$ & $\begin{array}{c}\mathrm{Pp}+10 \% \mathrm{TC} \\
+27,50 \% \mathrm{GPr} \\
+\mathrm{Ur}\end{array}$ & $\begin{array}{c}\mathrm{Pp}+30 \% \mathrm{FN} \\
+13 \% \mathrm{GPr} \\
+\mathrm{Ur}\end{array}$ \\
\hline & Ration 1 & Ration 2 & Ration 3 & Ration 4 & Ration 5 \\
\hline \multicolumn{6}{|l|}{ Coûts de production } \\
\hline \multicolumn{6}{|l|}{ Dépenses en liquidité } \\
\hline Aliments 1 & 2901 & 752 & 61 & 439 & 54 \\
\hline Animaux ${ }^{2}$ & 5796 & 6083 & 5733 & 6065 & 6184 \\
\hline Soins vétérinaires ${ }^{3}$ & 350 & 350 & 350 & 350 & 350 \\
\hline \multicolumn{6}{|l|}{ Dépenses en nature } \\
\hline Aliments 4 & 130 & 253 & 661 & 329 & 641 \\
\hline Coûts alimentation/charges (\%) & 33,0 & 13,5 & 10,6 & 10,7 & 9,61 \\
\hline \multicolumn{6}{|l|}{ Recettes } \\
\hline Recettes en liquidité 5 & 12342 & 10863 & 9147 & 10208 & 9900 \\
\hline Recettes en nature ${ }^{6}$ & 126 & 126 & 126 & 126 & 123 \\
\hline \multicolumn{6}{|l|}{ Résultats } \\
\hline Marges brutes a & 3291 & 3551 & 2468 & 3151 & 2797 \\
\hline Bénéfice net en liquidité b & 3295 & 3678 & 3003 & 3354 & 3312 \\
\hline Rapport coût-avantage ${ }^{c}$ & 1,36 & 1,48 & 1,36 & 1,44 & 1,39 \\
\hline Renouvellement capital d & 1,36 & 1,51 & 1,49 & 1,49 & 1,50 \\
\hline
\end{tabular}

Ksa : aliment Kibsa ; PS : paille de sorgho ; TC : tourteau de coton ; GPr : gousses de Piliostigma reticulatum ; Ur : urée ; Pp : foin de Pennisetum pedicellatum ; FN : foin de niébé

${ }^{1}$ Prix (Fcfa) par kilogramme de matière sèche $: \mathrm{Ur}=82 ; \mathrm{TC}=75 ; \mathrm{Ksa}=100 ;$ minéraux $=550$

${ }^{2}$ Prix moyen d'achat des animaux : 350 Fcfa par kilogramme de poids vif

${ }^{3}$ Coût moyen par animal : $350 \mathrm{Fcfa}$

${ }^{4}$ Coût estimé $:$ paille de sorgho $=7 \mathrm{Fcfa} ; \mathrm{GPr}=8 \mathrm{Fcfa} ; \mathrm{FN}=30 \mathrm{Fcfa} ; \mathrm{Pp}=9 \mathrm{Fcfa}$

5 Prix moyen réel à la vente : $550 \mathrm{Fcfa}$ par kilogramme de poids vif

${ }^{6}$ Fumier : $300 \mathrm{~g}$ par animal/j ; 5 Fcfa par kilogramme de fumier

a Recettes totales - coûts totaux

${ }^{\mathrm{b}}$ Recettes en liquidité - dépenses en liquidité

${ }^{c}$ Recettes totales / coûts totaux

${ }^{\mathrm{d}}$ Recettes en liquidité / dépenses en liquidité 
des fourrages pauvres. Les résultats ont également indiqué que, pour les besoins de production saisonnière de viande ovine dans ce contexte à faible disponibilité en capital financier, la combinaison entre une source azotée protéique (TC ou Ksa) à 20-30 p. 100 et un mélange GPr + Ur à 27 p. 100 permettait, d'une part, une utilisation optimale des fourrages pauvres (PS ou Pp) et, d'autre part, aux producteurs d'enregistrer les meilleurs gains économiques.
Les résultats ont toutefois révélé des limites liées à la mobilisation de sources azotées et énergétiques de bonne valeur au sein de la ferme en vue d'une substitution partielle ou totale des concentrés produits hors ferme. Ainsi, l'investissement dans la recherche de sources énergétiques et azotées de bonne valeur nutritive, telles les gousses d'essences légumineuses fourragères, apparaît justifié et permettrait de réduire de manière significative les coûts en liquidité tout en assurant un meilleur crôit pondéral.

\section{BIBLIOGRAPHIE}

1. AL-JASSIM R.A.M., EREIFEJ K.I., SHIBLI R.A., ABUDABOS A., 1998. Utilization of concentrate diets containing acorns (Quercus aegilops and Quercus coccifera) and urea by growing Awassi lambs. Small Ruminant Res., 29: 289-293.

2. AMEGEE Y., 1984. Le mouton de Vogan (croisé Djallonké x Sahélien) au Togo. III. Performances d'engraissement et rendements des carcasses. Revue Elev. Méd. vét. Pays trop., 37 : 97-106.

3. AMIR P., KNIPSCHEER H.C., 1989. Conducting on-farm animal research: Procedures and economics analysis. Arlington, VA, USA, Winrock International Institute for Agricultural Development/Ottawa, Canada, International Development Research Centre, 244 p.

4. BOCCARD R., DUMONT B.L., 1955. Etude de la production de viande chez les ovins. 1. La découpe des carcasses. Définition d'une coupe de référence. Ann. Zootech., $3:$ 241-257.

5. BOCCARD R., DUMONT B.L., 1960. Etude de la production de viande chez les ovins. II. Variation de l'importance des différentes régions corporelles de l'agneau de boucherie. Ann. Zootech., 9 : 356-363.

6. BOUGOUMA-YAMEOGO V., 1997. Influence de la qualité du fourrage et du taux de concentré sur les performance de croissance et d'engraissement de béliers "Djallonké » de type « Mossi ». Revue Méd. vét., 148 : 299-306.

7. BOURZAT D., BONKOUNGOU E. , RICHARD D., SANFO R., 1987. Essais d'intensification de la production animale en zone sahélosoudanienne : alimentation intensive de jeunes ovins dans le nord du Burkina. Revue Elev. Méd. vét. Pays trop., 40 : 151-156.

8. CELIK B., ALARSLAN O.F., 1998. A research on the effects of only barley ration and added urea as protein source on the fattening performance of lambs. Lalahan Hayvancilik Arastirma Enstitusu Dergisi, 38: $41-48$.

9. CONWAY E.J., 1962. Microdiffusion analysis and volumetric error, 5th Ed. London, UK, Crosby Lockwood, 468 p.

10. DEHOUX J.P., HOUNSOU-VE G., 1991. Essai préliminaire d'embouche intensive de béliers Fulani et Djallonké à base de céréales (mil et maïs) et de graines de coton, au nord-est du Bénin. Tropicultura, 9: 151-154

11. GARCIA C.A., DA SILVA-SOBRINHO A.G., FERNANDES E.A., SCHENCK P.F., 1995. The use of urea as partial cotton meal substitution for lambs in growth. UNIMAR Ciencias, 4: 42-48.

12. GRYSEELS G., 1988. Role of livestock on mixed smallholder farms in the Ethiopian Highlands: A case study from Baso and Worena Wereda near Debre Berhan. PhD thesis, Agricultural University, Wageningen, The Netherlands, $249 p$.

13. KABBALI A., JOHNSON W.L., GOODRICH R.D., ALLEN C.E., 1992. Effect of compensatory growth on some body component weights and on carcass and non carcass composition of growing lambs. J. Anim. Sci., 70: 2852-2858.

14. KABBALI A., JOHNSON W.L., GOODRICH R.D., ALLEN C.E., 1992. Effect of under-nutrition and refeeding on weights of body parts and chemical components of growing Moroccan lambs. J. Anim. Sci., 70: 2859-2865.

15. KHAMIS H.S., EL SAHER H.M., SALEM O.A., 1996. Fattening sheep on diets of broiler litter ensiled with green Berseem (Trifolium alexandrinum L.): Nutritional evaluation of fattening sheep diets. Small Ruminant Res. Network Newsl. (32): 4-8.

16. NDEMANISHO E.E., MTENGA L.A., KIMBI E.F.C., KIMAMBO A.E., MTENGETI E.J., 1998. Substitution of dry Leucaena leucocephala (DDL) leaves for cotton seed cake (CSC) as a protein supplement to urea treated maize stover fed to dairy weaner goats. Anim. Feed Sci. Technol., 73: 365-374.

17. NIANOGO A.J., LOUIS S.L., SOLAIMAN S., OUEDRAOGO C.L., SIAWAY A., 1999. Effect of urea treatment on digestibility and utilization of sorghum straw. Biotechnol., Agron., Soc. Environ. (BASE), 3: 78-85.

18. NIANOGO A.J., NASSA S., SOMA L., SANON H.O., 1997. Influence de la complémentation et du mode de conduite sur la croissance des agneaux Mossi en saison pluvieuse. Bull. Anim. Health Prod. Afr., 45 : 241-249.

19. NIANOGO A.J., SOMA L., BONKOUNGOU G.F.X., NASSA S., ZOUNDI S.J., 1995. Utilisation optimale de la graine de coton et des fourrages locaux pour l'engraissement des ovins Djallonké type Mossi. Revue Rés. Amélior. Prod. agr. Milieu aride, 7 : 179-195.

20. PRESTON T.R., LENG R.A., 1986. Matching livestock production systems to available resources (pre-testing edition). Addis Ababa, Ethiopia, ILCA, $331 \mathrm{p}$.

21. RIVIERE R., 1991. Manuel d'alimentation des ruminants domestiques en milieu tropical. Paris, France, ministère de la Coopération et du développement, $529 \mathrm{p}$.

22. SAS, 1982. User's guide. Cary, NC, USA, SAS Institute, $40 \mathrm{p}$.

23. SAVADOGO M., 2000. Crop residue management in relation to sustainable land use. A case study in Burkina Faso. PhD thesis, Agricultural University, Wageningen, The Netherlands, 159 p.

24. TAMBOURA H., ABOU F., 1992. Utilisation des blocs mélasse-urée pour la valorisation des pailles de riz dans l'alimentation des moutons en pays tropicaux sahéliens. Bull. Anim. Health Prod. Afr., 40 : 25-31.

25. TAREQUE A.M.M., 1987. Non-protein and agro-industrial byproducts utilization by ruminants in Bangladesh. In: Proc. Final coordination meeting "Isotope aided studies on non-protein nitrogen and agro-industrial by-products utilization by ruminants", Vienna, Austria, 24-26 March 1986. Vienna, Austria, FAO/IAEA, p. 129-142.

26. THYS E., 1991. L'utilisation de tourteau et de coques de coton à haute dose dans I'alimentation de béliers de I'extrême Nord Cameroun. Observations préliminaires. Tropicultura, 4 : 132-136.

27. TIENDREBEOGO J.-P., 1992. Embouche ovine améliorée. Etude comparée des différentes rations alimentaires à forte proportion de fourrages naturels locaux. Revue sci. tech., $20: 68-78$.

28. ZOUNDI S.J., NIANOGO A.J., SAWADOGO L., 1996. Utilisation de gousses de Piliostigma reticulatum (DC.) Hochst. et de feuilles de Cajanus cajan (L.) Millsp. en combinaison avec l'urée pour I'engraissement de moutons Djallonké type Mossi et du Sud au Burkina. Tropicultura, 14 : 149-152.

29. ZOUNDI S.J., SAWADOGO L.L., NIANOGO A.J., 1994. Croissance compensatrice d'ovins alimentés sur parcours naturels : analyse des grains de poids vif et des caractéristiques de la carcasse. Revue Rés. Amélior. Prod. agr. Milieu aride, 6 : 179-196.

Reçu le 30.11.2000, accepté le 13.12.2002 


\section{Summary}

Zoundi J.S., Nianogo A.J., Sawadogo L. Optimal Use of Locally Available Feed Resources for Sheep Fattening in Mixed Crop-Livestock Farming Systems in the Central Plateau of Burkina Faso

Two trials were carried out on Djallonke fattening sheep to investigate feed systems that would produce meat at lower cost. "Kibsa Aliment" (KSA), a commercial concentrate (17.60\% digestible crude protein, $0.91 \mathrm{UF} / \mathrm{kg}$ dry matter), or cottonseed cakes (CSC) were partially or totally substituted by local energy and nitrogen sources. A control diet (D1) [40\% sorghum straw (SS) $+60 \% \mathrm{KSA}$ ] was thus compared to various feed combinations that included local products: in D2, KSA was partially substituted $(-30 \%)$ by $27.50 \%$ Piliostigma reticulatum pods $(\mathrm{PrP})+$ urea $(\mathrm{Ur})$, and in $\mathrm{D} 3, \mathrm{KSA}$ was totally substituted by $47 \%$ CSC $+\operatorname{PrP}+$ Ur. In trial 1 , the average daily gains $(A D G)$ were significantly different $(P<0.01)$ with values of 100.5, 62.4, and 58.6 g recorded in D1, D2, and D3, respectively. These substitutions led to ADG losses of - 38.1 and - $49.2 \mathrm{~g}$, respectively. However, D2 and D3 yielded the highest profits with extra benefits of +519 and +1055 CFA francs net per animal. In trial 2, CSC was partially substituted $(-10 \%)$ by Pennisetum pedicellatum (Pp) hay, which resulted in ADG losses of - $14.4 \mathrm{~g}$. In conclusion, the combination of $20-30 \%$ CSC or KSA, and $27 \% \operatorname{PrP}+\mathrm{Ur}$ enhanced the fodder (SS or Pp) quality and enabled producers to register the highest profits.

Key words: Djallonke sheep - Fattening - Substitute feed Urea - Piliostigma reticulatum - Cost benefit analysis Burkina Faso.

\section{Resumen}

Zoundi J.S., Nianogo A.J., Sawadogo L. Uso optimizado de los recursos alimenticios disponibles localmente para el engorde de ovinos en el seno de explotaciones mixtas agricultura-cría en la meseta central de Burkina Faso

Se llevaron a cabo ensayos con los ovinos Djallonke de engorde, con el fin de buscar los sistemas de alimentación para la producción de carne a menor costo. Durante el presente estudio se examinaron las posibilidades de substitución parcial o total de un concentrado comercial "Alimento Kibsa" (Ksa) $(17,60 \%$ de materias nitrogenadas digeribles; 0,91 UF/kg de materia seca) o torta de algodón (TC) mediante fuentes energéticas y nitrogenadas disponibles localmente. De esta manera, se comparó una ración testigo (R1) [40\% paja de sorgo (PS) $+60 \% \mathrm{Ksa}$ ] con diferentes combinaciones alimenticias integrando productos locales: en R2 hubo una substitución parcial de Ksa (-30\%) por $27,50 \%$ de vainas de Piliostigma reticulatum $(\mathrm{GPr})$ + urea $(\mathrm{Ur})$ y en $\mathrm{R} 3$ hubo una substitución total de Ksa por $47 \%$ de TC + GPr + Ur. Las ganancias medias diarias (gmq) registradas durante la prueba 1 presentaron diferencias significativas $(P<0,01)$ con 100,5 , 64,2 y 58,6 g respectivamente para R1, R2 y R3. Estas substituciones se traducen por pérdidas respectivas de gmq de - 38,1 y de - 49,2 g. Sin embargo, R2 y R3 presentaron mejores ganancias, con beneficios respectivos netos adicionales de +519 y +1055 Fcfa por animal. El estudio 2 mostró que la substitución parcial de TC $(-10 \%)$ por heno de Pennicetum pedicellatum (Pp) se traduce por pérdidas de gmq de $-14,4$ g. En conclusión, la combinación de entre 20-30\% de TC o de Ksa y de $27 \%$ de GPr + Ur condujo a una buena valorización de los forrages (PS o Pp) y permitió a los productores mejores ganancias económicas.

Palabras clave: Ovino Djallonke - Engorde - Alimento sustitutivo - Urea - Piliostigma reticulatum - Analisis de costos y beneficios - Burkina Faso. 